\title{
Interprétation des concentrations sanguines en cannabinoïdes : données actuelles
}

\section{Interpretation of cannabinoids levels in blood : current data}

\section{Patrick MURA*, Bertrand BRUNET, Carla MONZON, Yves PAPET, Gérard MAUCO}

(1) Laboratoire de Biochimie et Toxicologie, Centre Hospitalier Universitaire - BP 577 - 86021 POITIERS

* Auteur à qui adresser la correspondance : Patrick MURA, Laboratoire de Biochimie et Toxicologie BP 577 - 86021 POITIERS - Tél : (33) 549443923 - E-mail : p.mura@chu-poitiers.fr

\section{RÉSUMÉ}

Le cannabis est le produit psychoactifillicite le plus consommé en France et dans de nombreux autres pays. Les effets chez l'homme sont principalement dus au $\Delta 9$-tétrahydrocannabinol (THC) et au 11-OH- -9 -tétrahydrocannabinol (11$\mathrm{OH}-\mathrm{THC}$ ) et sont responsables de nombreux problèmes de santé publique, tels que les accidents de la voie publique, les accidents du travail, le dopage ou la soumission chimique. C'est pour toutes ces raisons que le dosage des cannabinoïdes est de plus en plus pratiqué dans les laboratoires de toxicologie. Les techniques analytiques sont désormais très performantes. Les concentrations sanguines reflètent-elles le degré d'altération des fonctions cognitives et motrices d'un individu? S'il existe une bonne corrélation entre la dose et les effets, la corrélation entre dose et concentration sanguine existe uniquement au moment du pic sanguin. En raison d'une très forte et rapide fixation tissulaire, aucune corrélation n'a été observée entre les concentrations sanguines en cannabinoüdes et les effets, qui sont à leur apogée alors que les concentrations en principes actifs sont devenues très faibles. La connaissance des concentrations sanguines en THC et/ou 11-OH-THC ne permet donc pas d'apprécier le degré d'altération des performances cognitives et motrices et la notion de seuil de dangerosité pour la concentration

\section{SUMMARY}

Cannabis is the major drug of abuse consumed in France as well as in many other countries. Effects in human are mainly due to $\Delta 9$-tetrahydrocannabinol (THC) and 11-OH- $\triangle 9$ tetrahydrocannabinol (11-OH-THC). These effects are involved in many public health problems such as traffic safety, workplace accidents, doping or drug facilitated assaults. Therefore, identification and determination of cannabinoids in blood is increasingly performed in toxicology laboratories. Analytical procedures are now highly efficient. Do blood concentrations of cannabinoids reflect the degree of impairment of cognitive and psychomotor performances? Whereas a good correlation is observed between doses and effects, the correlation between doses and blood levels of cannabinoids only exists at the moment of the blood concentration peak. Because of a very quick and strong distribution in fat tissues, no correlation could be observed between blood levels of cannabinoids and the effects, which reach their maximum when blood concentrations in active analytes become very low. Consequently, blood concentrations in THC and/or 11-OH-THC do not allow the degree of impairment of cognitive and psychomotor performances to be evaluated. Consequently, the determination of a blood level risk cut-off is not scientifically conceivable. It is the reason why, 
sanguine n'est donc pas envisageable. C'est pourquoi, dans l'attente de travaux complémentaires, la seule mise en évidence de principes actifs du cannabis dans le sang au dessus d'un seuil analytique préalablement défini permet d'affirmer que le sujet a récemment consommé du cannabis, et donc de pouvoir estimer que le sujet était sous influence de cannabis au moment du prélèvement.

\section{MOTS-CLÉS}

Cannabis, THC, concentrations sanguines, accidents, soumission chimique, interprétation.

\section{Introduction}

Parmi les substances illicites, le cannabis est de loin le produit le plus utilisé en France comme dans la plupart des pays. Ainsi, une récente enquête en France a montré qu'à l'âge de 18 ans, 55,7 \% des garçons et 45,2\% des filles avaient déjà expérimenté le cannabis et qu'environ $20 \%$ des garçons en consommaient plus de 10 fois par mois (1).

Les risques liés à l'usage de cannabis sont nombreux. A côté des risques somatiques (cardio-vasculaires, broncho-pulmonaires, digestifs, sur les fonctions de reproduction ou sur le système immunitaire) (2), ce sont essentiellement les risques induits par les effets psychiques qui dominent (3). En effet, le cannabis se présente comme un produit aux effets psychotropes majeurs. Diminution de la vigilance, modifications de la perception du temps et des distances, rétrécissement du champ visuel, perturbations de la mémoire à court terme, troubles thymiques et dissociatifs avec euphorie, dépersonnalisation avec disparition des inhibitions sont autant d'éléments pouvant avoir des conséquences très graves dans de nombreuses circonstances de la vie quotidienne. Une étude récente a ainsi montré que, chez les conducteurs ayant moins de 27 ans, la fréquence des accidents corporels de la voie publique était multipliée par 2,5 après un usage récent de cannabis (4). En milieu professionnel, plusieurs travaux ont révélé que les consommateurs représentaient une menace réelle pour les entreprises (5-7). Dans le cadre de la soumission chimique, le cannabis peut être utilisé à des fins criminelles (8).

C'est pour toutes ces raisons que le dosage des cannabinoïdes dans le sang occupe une part de plus en plus importante dans l'activité des toxicologues analystes.

$\mathrm{Si}$ les méthodes analytiques sont désormais très fiables (9), permettant de détecter la présence de cannabinoïdes à des concentrations inférieures au ng/ml, l'interprétation des résultats a été l'objet de très peu d'études. Pourtant, il est essentiel que le toxicologue puisse répondre à cette question si souvent posée par les magistrats : au vu des concentrations en cannabi- looking forward to complementary studies, the unique presence of active metabolites of cannabis in blood above an analytical threshold previously defined attests of a recent use of cannabis which allows to consider that the subject was under the influence of cannabis at the moment of blood sampling.

\section{KEY-WORDS}

Cannabis, THC, blood concentrations, accidents, chemical submission, interpretation.

noïdes, le sujet était-il ou non sous influence au moment des faits ? En d'autres termes, l'usage de cannabis peut-il être considéré comme ayant été l'élément responsable de l'accident ou du délit?

\section{Relation doses - effets}

Barnett et coll (10) ont étudié, sur simulateurs de conduite, les effets obtenus chez 8 volontaires masculins par la consommation de «joints» dosés à 100, 200 ou $250 \mu \mathrm{g} / \mathrm{kg}$ de poids corporel. Les temps de réponse aux évènements augmentaient avec les doses, de même que le nombre d'erreurs de conduite.

Cette bonne corrélation entre les doses et les effets a été confirmé par les travaux de Harder et coll. (11) et Cochetto et coll. (12), en comparant les effets «ressentis» par des sujets ayant fumé des joints contenant 1, 3 et $9 \mathrm{mg}$ de THC.

Il est à noter toutefois que cette corrélation existe pour un sujet donné, mais pas entre les individus. En effet, il est bien établi que les effets sont très variables d'un individu à l'autre et selon la manière de fumer (3).

\section{Relation doses - concentra- tions sanguines en THC}

15 à $50 \%$ du THC présent dans la fumée d'un «joint» sont absorbés par voie respiratoire et passent dans le flux sanguin. Cette absorption est très rapide et les concentrations sanguines maximales (50 à $300 \mathrm{ng} / \mathrm{ml}$ ) sont obtenues en 7 à 10 minutes après le début de l'inhalation, comme le montre la Figure 1 (13). Au moment du pic, les concentrations sont dose-dépendantes. Très lipophile, le THC se distribue rapidement dans tous les tissus riches en lipides dont le cerveau. Le volume de distribution dans l'organisme est de 4 à $14 \mathrm{~L} / \mathrm{kg}$. Cette fixation tissulaire importante est responsable d'une diminution rapide des concentrations sanguines. Deux heures après le début de l'inhalation d'un «joint» contenant $1,75 \%$ de THC, les concentrations sanguines sont inférieures à $5 \mathrm{ng} / \mathrm{ml}$ (14) et restent 
détectables pendant 8 à 12 heures. A ce stade, aucune corrélation dose-concentration sanguine en THC ne peut plus être observée, comme l'illustre la Figure 2.

En résumé, pour un même individu, une corrélation dose-concentration sanguine est observable uniquement au moment du pic plasmatique (avec une très grande variabilité inter-individuelle).

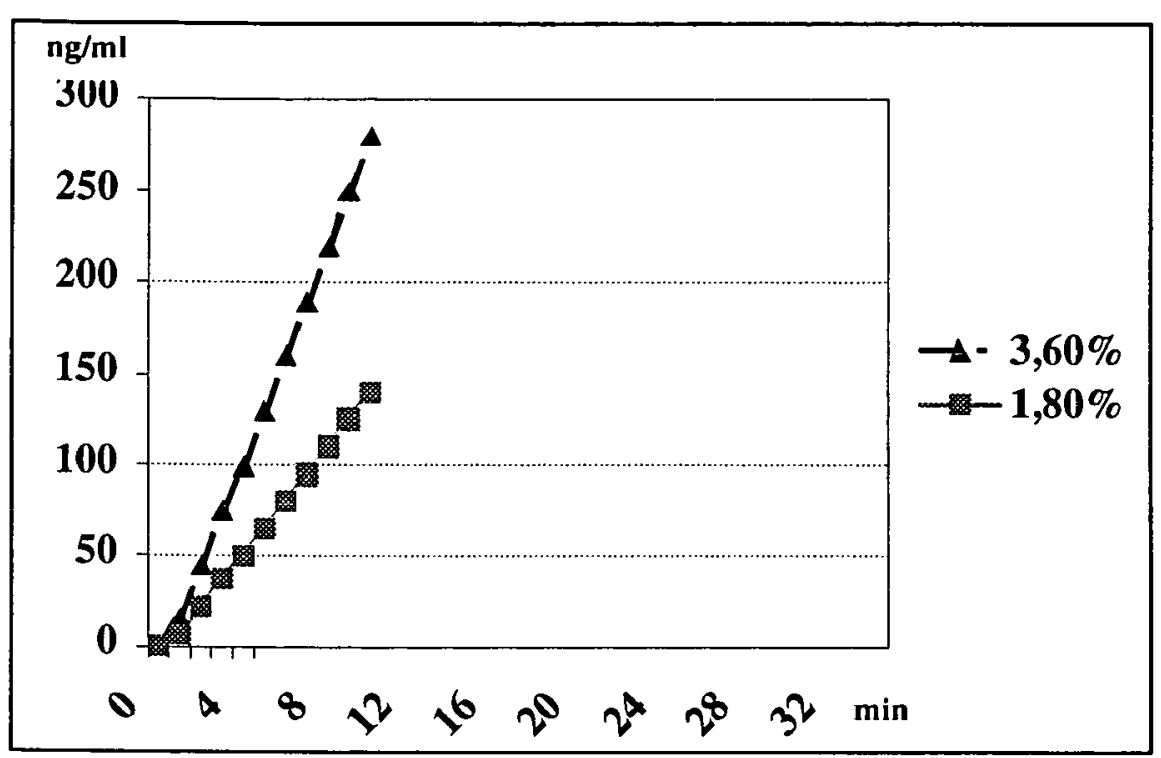

Figure 1 : Augmentations des concentrations sanguines en THC après consommation d'un "joint" contenant $1,80 \%$ de THC (carrés) ou 3,60 \% de THC (triangles). Chaque flèche représente une inhalation.

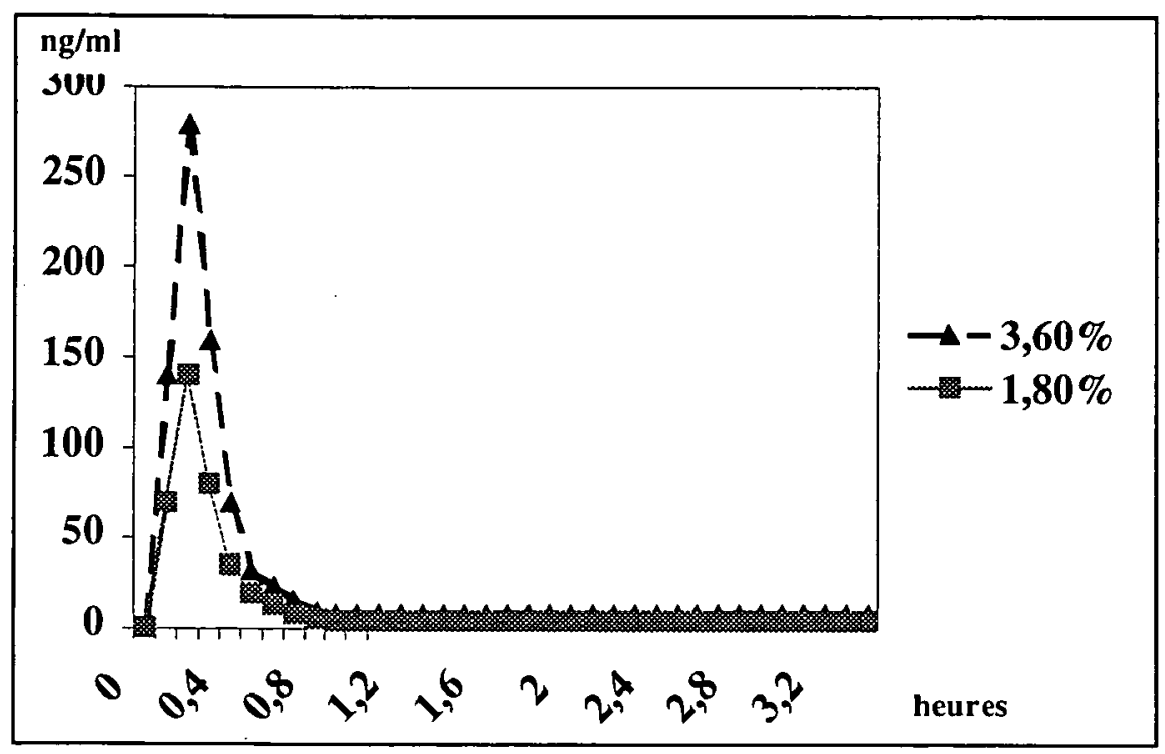

Figure 2 : Évolutions comparés des concentrations sanguines en THC après consommation d'un "joint" contenant 1,80\% de THC (carrés) ou 3,60\% de THC (triangles).

\section{Relation concentrations sanguines en THC - effets}

La très forte lipophilie du THC, l'existence d'un cycle entéro-hépatique et de la réabsorption rénale se traduisent par des effets psychoactifs prolongés (15).

Sur la base de travaux antérieurs (12), Harder et coll (13) ont comparé l'évolution dans le temps des concentrations sanguines de THC et les effets psychiques res- sentis par les sujets, après consommation de «joints» contenant $9 \mathrm{mg}$ de THC (joint standard» tel qu'il est défini aux États-Unis par le National Institute on Drug Abuse), $3 \mathrm{mg}$ ou $1 \mathrm{mg}$. Les résultats, illustrés par la Figure 3, montrent que les effets psychiques obtenus après consommation isolée d'un joint contenant $9 \mathrm{mg}$ de THC persistent pendant une durée d'environ $2 \mathrm{~h}$, tandis que la concentration en THC dans le sang est rapidement très faible et de l'ordre $\mathrm{du} \mathrm{ng} / \mathrm{ml}$ au bout de $2 \mathrm{~h}$. Ces observations ont été confirmées par Huestis et coll. (16).

Johansson et coll. (17) ont détecté du delta-9-tétrahydrocannabinol dans des biopsies de tissus adipeux 4 semaines après la dernière consommation de cannabis. Bien que les corrélations entre concentrations sanguines et concentrations cérébrales en cannabinoïdes n'aient pas été l'objet d'études à ce jour, il est logique de supposer que des effets soient encore présents alors que les concentrations sanguines sont devenues très faibles.

Toujours est-il qu'en raison des caractéristiques toxicocinétiques des principes actifs du cannabis et contrairement à ce qui est observé avec l'alcool, les concentrations sanguines en cannabinoïdes ne peuvent pas être utilisées pour préjuger des effets psychoactifs chez le consommateur. Cela a été confirmé récemment par une étude réalisée en France chez' 900 conducteurs impliqués dans un accident corporel de la voie publique et dont les résultats ont été comparés avec 900 sujets témoins (4). Au cours de cette étude, les auteurs ont montré que, si la fréquence des accidents chez les moins de 27 ans ayant récemment consommé du cannabis était multipliée par 2,5 (odds-ratio $=2,5$ ), ce risque était sensiblement identique selon que les concentrations sanguines en THC étaient basses ou élevées.

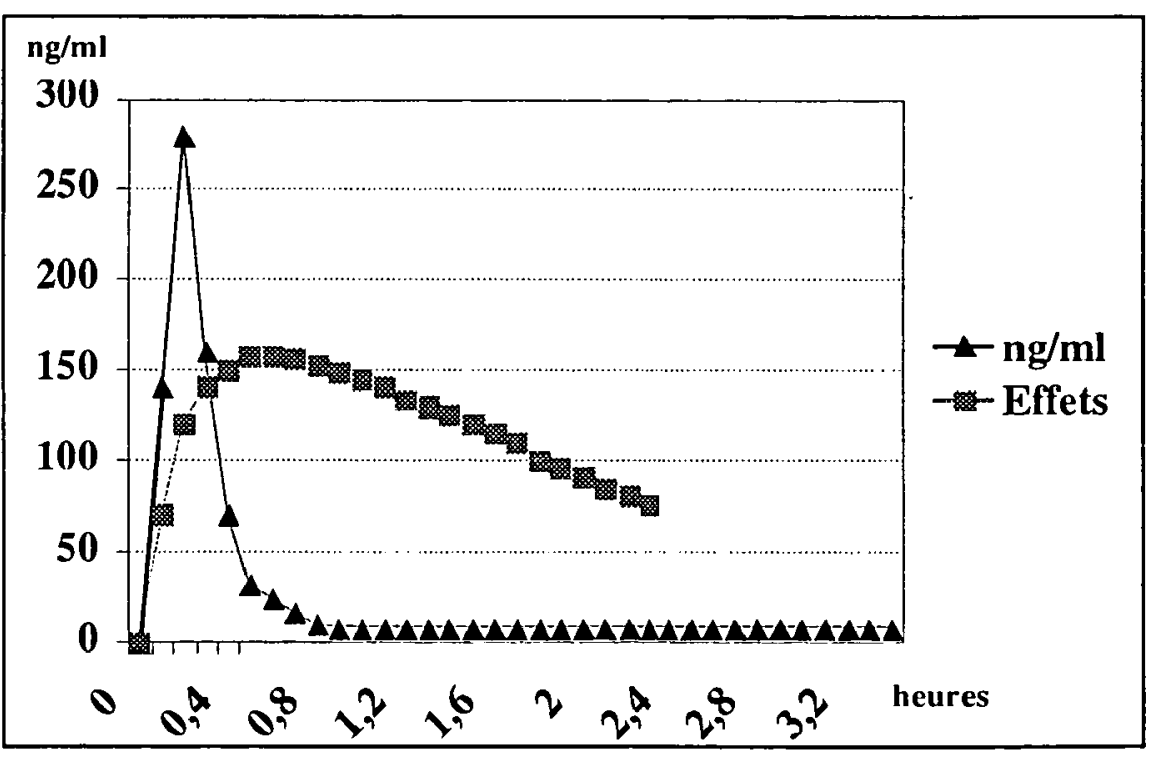

Figure 3 : Concentrations en THC (triangles) et effets physiques et psychiques "ressentis" par le sujet (carrés) en fonction du temps, après consommation d'un "joint" contenant $9 \mathrm{mg}$ de THC. 


\section{Rapports de concentrations entre les différents cannabi- noïdes}

Le THC subit un métabolisme oxydatif conduisant aux principaux composés suivants :

- le 11-hydroxy-tétrahydrocannabinol (11-OH THC). Il s'agit d'un métabolite psychoactif. Ses concentrations sanguines sont généralement de 4 à $20 \mathrm{ng} / \mathrm{ml}$ après 20 minutes et inférieures à $1 \mathrm{ng} / \mathrm{ml} 4$ heures après le début de l'inhalation.

- le 11-nor-9-carboxy- $\Delta$ 9-tétrahydrocannabinol (THC$\mathrm{COOH})$. Obtenu par oxydation du 11-OH THC, il ne possède aucune activité pharmacologique. Cet acide commence à apparaître dans le sang dans les minutes qui suivent l'inhalation. Au cours des étapes successives de distribution et de métabolisme du THC, les concentrations en THC-COOH dans le sang augmentent tandis que celles de THC décroissent.

Huestis et coll. ont déterminé deux modèles mathématiques prenant en compte les concentrations plasmatiques en THC et en THC-COOH, permettant d'estimer le délai entre l'inhalation et le prélèvement (18). Bien que n'ayant pas été validé par d'autres auteurs, ce modèle serait applicable dans le cas d'une consommation isolée mais n'est en aucun cas utilisable pour des consommations répétées. En effet, du fait de la rémanence importante des cannabinoïdes dans les tissus lipidiques de l'organisme, les concentrations sanguines en THC-COOH sont beaucoup plus élevées chez les consommateurs réguliers que chez les consommateurs occasionnels (19). Par ailleurs, une étude récente (20) a montré que les modèles de Huestis ayant été déterminés chez des sujets vivants à partir de concentrations dans le plasma, il n'est pas possible de les utiliser dans le cas d'échantillons de sang post-mortem en raison de la redistribution post-mortem des cannabinoïdes.

Daldrup (21) a proposé une formule combinant les concentrations en THC, 11-OH THC et THC-COOH et conduisant à l'établissement du «Cannabis Influence Factor » pour lequel une valeur supérieure à 10 traduit la présence d'effets pharmacologiques. Cependant, cette étude n'a, à ce jour, jamais été validée par d'autres auteurs.

De plus, lorsque le cannabis est consommé par ingestion, la quasi-totalité du THC est hydroxylée (principalement en 11-OH THC) au niveau de la muqueuse intestinale, ce qui se traduit dans le compartiment sanguin par une concentration en 11-OH THC supérieure à celle du $\mathrm{THC}$, contrairement à ce qui est observé lorsque le cannabis est inhalé.

\section{Durées des effets et de la détectabilité des cannabi- noïdes dans le sang}

Après inhalation de THC, le THC-COOH peut être détecté dans le sang pendant de nombreuses heures (48 à 168 h). En revanche, la durée pendant laquelle les principes actifs (THC et 11-OH-THC) peuvent être retrouvés à concentration significative (supérieure à $0,5 \mathrm{ng} / \mathrm{ml}$ ) dans le sang est relativement courte, étant au maximum de l'ordre de 4 à $5 \mathrm{~h}$ pour le $11-\mathrm{OH}-\mathrm{THC}$ et variant entre 3 à $12 \mathrm{~h}$ pour le THC $(16,22)$.

Selon la plupart des auteurs, les effets physiologiques et comportementaux disparaissent dans les 3 à $6 \mathrm{~h}$ après exposition. Quelques auteurs ont cependant rapporté des effets résiduels sur le comportement pouvant persister pendant $24 \mathrm{~h}(23,24)$.

On observe donc une certaine similitude entre la période pendant laquelle les principes actifs du cannabis sont détectables dans le sang et le temps pendant lequel les consommateurs peuvent être considérés comme étant sous influence de cannabis.

\section{Seuil de dangerosité et seuil analytique}

Du fait de ces propriétés toxicocinétiques, les concentrations sanguines en cannabinoïdes ne permettent donc pas de préjuger du degré de l'altération comportementale induite par la consommation de cannabis. En conséquence et à fortiori, il s'avère illusoire et sans fondement scientifique de vouloir déterminer un seuil sanguin de dangerosité, en particulier dans le cadre de la sécurité routière.

En revanche, il est indispensable de fixer un seuil analytique, en dessous duquel la recherche de cannabinoïdes serait considérée comme négative. Ce seuil est à déterminer en prenant en compte les performances techniques (sensibilité, spécificité, reproductibilité) des laboratoires pratiquant ce genre d'analyses. En France et sur la base des résultats d'un contrôle de qualité organisé chaque année par la Société Française de Toxicologie Analytique (25), il est désormais possible de proposer un seuil de $0,5 \mathrm{ng} / \mathrm{ml}$ pour chacun des cannabinoïdes.

\section{Conclusion}

Il serait évidemment très utile que les concentrations sanguines en cannabinoïdes puissent être utilisées pour 
juger des altérations comportementales induites par une consommation de cannabis. Cependant, la présence de plusieurs principes actifs, le manque de corrélation entre les concentrations sanguines et les effets ainsi que les variations de l'importance relative des différentes voies métaboliques selon les voies d'administration ou selon qu'il s'agisse de consommations. isolées ou répétées sont autant d'éléments interdisant toute tentative d'interprétation. La période de détectabilité des principes actifs dans le sang étant superposable à celle des effets, la seule alternative restant à ce jour est, dans l'attente de travaux complémentaires, de considérer que la seule présence dans le sang de THC et/ou 11-OH-THC au dessus d'un seuil analytique préalablement défini (et proposé à $0,5 \mathrm{ng} / \mathrm{ml}$ ) permet d'affirmer que le sujet a récemment consommé du cannabis et donc d'estimer qu'il était sous influence de cannabis au moment du prélèvement.

\section{Références}

1. ESCAPAD 2001. Santé, mode de vie et usages de drogues à 18 ans. OFDT, Paris, juin 2002.

2. Expertise collective INSERM. Cannabis. Quels effets sur le comportement et la santé ? In : INSERM, ed. Paris : Jouve, 2001.

3. Mura P., Piriou A. Cannabis. In : Kintz P ed. Toxicologie et pharmacologie médicolégales. Elsevier, Paris, 1998, pp. 543-554.

4. Mura P., Kintz P., Ludes B., Gaulier J.M., Marquet P., Martin-Dupont S., Vincent F., Kaddour A., Goullé J.P., Nouveau J., Moulsma M., Tilhet-Coartet S., Pourrat O. Comparison of the prevalence of alcohol, cannabis and other drugs between 900 injured drivers and 900 control subjects : results of a French collaborative study. Forensic Sci. Int. 2003 (in press).

5. Kandel D.B., Davies M. Labor force experiences of a national sample of young adult men. Youth and Society. $1990 ; 21: 411-445$.

6. Carter W.E., Doughty P. Social and cultural aspects of cannabis use in Costa Rica. Ann N Y Acad Sci. 1976 ; $282: 2-16$.

7. Mura P. Cannabis sativa var. indica : une menace croissante pour les entreprises. Annales de Toxicologie Analytique $2002 ; 14: 64-67$.

8. Mura P., Visinoni P., Alvarez J.C., Goullé J.P., Kintz P. Le cannabis : quelle place dans la soumission chimique ? Annales de toxicologie Analytique 2002 ; 14 : 412-416.

9. Kintz P., Cirimele V., Pepin G., Marquet P., Deveaux M., Mura P. Identification et dosage des cannabinoïdes dans le sang total. Toxicorama $1996 ; 8: 29-33$.

10. Barnett G., Licko V., Thompson T. Behavioral pharmacokinetics of marijuana. Psychopharmacology 1985 ; $85: 51-56$.
11. Harder S., Rietbrock S. Concentration-effect relationship of (-9-tetrahydrocannabinol and prediction of psychotropic effects after smoking marijuana. International Journal of Clinical Pharmacology and Therapeutics 1997 ; 35 : 155-159.

12. Cochetto D.M., Owens S.M., Perez-Reyes M., DiGuiseppi S., Miller L.L. Relationship between plasma delta-9-tetrahydrocannabinol concentration and pharmacologic effects in man. Psychophamacology $1981 ; 75$ : 158-164.

13. Huestis M.A., Sampson A.H., Holicky B.J., Henningfield J.E., Cone E.J. Characterization of the absorption phase of marijuana smoking. Clin Pharmacol Ther. 1992 ; 52 : 31-41.

14. Huestis M.A., Henningfield J.E., Cone E.J. Blood cannabinoids. I. Absorption of THC and formation of 11-OHTHC and THCCOOH during and after smoking marijuana. J Anal Toxicol $1992 ; 16: 276-282$.

15. Stillman R., Galanter M., Lemberger L. Tetrahydrocannabinol : metabolism and subjective effects. Life Sci $1976 ; 19: 569-576$.

16. Huestis M.A. Cannabis - effects on human behavior and performance. Forensic Sci. Rev. 2002 ; 14 : 15-59.

17.Johansson E., Noren K., Sjovall J., Halldin M.M. Determination of delta-1-tetrahydrocannabinol in human fat biopsies from marihuana users by gas chromatography-mass spectrometry. Biomed. Chromatogr. $1989 ; 3$ : 35-38.

18. Huestis M.A., Henningfield J.E., Cone E.J. Blood cannabinoids. II. Models for the prediction of time of marijuana exposure from plasma concentrations of Delta9- tetrahydrocannabinol and 11-nor-9-carboxy-Delta9- tetrahydrocannabinol. J. Anal. Toxicol. $1992 ; 16: 283-290$.

19. Kelly P., Jones R.T. Metabolism of tetrahydrocannabinol in frequent and infrequent marijuana users. J. Anal. Toxicol. $1992 ; 16: 228-235$.

20. Giroud C., Ménétrey A., Augsburger M., Buclin T., Sanchez-Mazas P., Mangin P. $\Delta 9$-THC, 11-OH- $\Delta 9-\mathrm{THC}$ and $\triangle 9-\mathrm{THCCOOH}$ plasma or serum to whole blood concentrations distribution ratios in blood samples taken from living and dead people. Forensic Sci. Int. 2001 ; $123: 159-164$.

21.Daldrup T. Cannabis influence factor. Congrès de l'International Association of Forensic Sciences, Tokyo, 1996.

22. Verstraete A. Fenêtres de detection des xénobiotiques dans le sang, les urines, la salive et les cheveux. Annales de Toxicologie Analytique $2002 ; 14: 390-394$.

23. Heishman S.J., Huestis M.A., Henningfield J.E., Cone E.J. Acute and residual effects of marijuana: profile of plasma THC levels, physiological, subjective, and performance measures. Pharmacol Biochem Behav 1990 ; 37 : 561-565.

24. Leirer V.O., Yesavage J.A., Morrow D.G. Marijuana carry-over effects on aircraft pilot performance. Aviat. Space Eviron. Med. $1991 ; 62: 221-227$.

25. Deveaux M., Hedouin V., Marquet P., Kintz P., Mura P., Pepin G. Conduite automobile et stupéfiants : procédures de dépistage et de dosage, accréditation des laboratoires. Toxicorama $1996 ; 8: 11-15$. 